\title{
Trends in Meeting the Physical Activity Guidelines: Muscle-Strengthening Alone and Combined With Aerobic Activity, United States, 1998-2018
}

\author{
Eric T. Hyde, Geoffrey P. Whitfield, John D. Omura, Janet E. Fulton, and Susan A. Carlson
}

\begin{abstract}
Background: The National Health Interview Survey is unique among US federal surveillance systems with over 20 years of consistent assessment of muscle-strengthening and aerobic activity. The authors examined trends in the prevalence of US adults who met the muscle-strengthening ( 2 or more days per week) and the combined muscle-strengthening and aerobic physical activity (at least $150 \mathrm{~min} / \mathrm{wk}$ of moderate-intensity equivalent activity) guidelines from 1998 to 2018 . Methods: The 1998-2018 National Health Interview Survey data were analyzed. Age-adjusted prevalence of meeting the muscle-strengthening and combined aerobic and muscle-strengthening guidelines by selected respondent characteristics were estimated for each year and linear and higher-order trends were assessed. Results: From 1998 to 2018, prevalence of meeting the musclestrengthening guideline increased from $17.7 \%$ to $27.6 \%$, and meeting the combined aerobic and muscle-strengthening guidelines increased from $14.4 \%$ to $24.0 \%$. All subgroups demonstrated significant increases in meeting both guideline measures over this period although trends varied across the 21 years; increasing trends were more commonly sustained in the second decade of monitoring. Conclusions: Although increasing trends in prevalence of meeting the muscle-strengthening and combined guidelines are encouraging, current prevalence estimates remain low. Opportunities exist for the continued promotion of muscle-strengthening activity using evidence-based approaches.
\end{abstract}

Keywords: fitness, surveillance, NHIS, muscle-strengthening, guidelines

The past 2 editions of the Physical Activity Guidelines for Americans (Guidelines) have recommended that adults should do muscle-strengthening activities of moderate or greater intensity and that involve all major muscle groups on 2 or more days per week (muscle-strengthening guideline) for health benefits. ${ }^{1-3}$ The muscle-strengthening guideline is in addition to the minimal aerobic physical activity guideline, which recommends adults should do at least 150 minutes a week of moderate intensity, or 75 minutes a week of vigorous-intensity aerobic physical activity, or an equivalent combination of moderate- and vigorous-intensity aerobic activity (aerobic guideline) to obtain substantial health benefits. ${ }^{1,2}$ However, a number of health benefits provided by musclestrengthening activities, including increased bone strength, muscular fitness, and muscle mass retention during weight loss, are not found in aerobic activity alone. ${ }^{1-3}$

The National Health Interview Survey (NHIS) has been the primary data source used for monitoring progress toward achieving Healthy People physical activity objectives, such as the Healthy People 2010, 2020, and 2030 goals of increasing the proportion of adults meeting the muscle-strengthening and aerobic physical activity guidelines (separately and in combination). ${ }^{4-6}$ In addition, NHIS provides a unique opportunity to examine trends in the prevalence of meeting physical activity guidelines from 1998 to 2018 since the survey methodology and the physical activity

Hyde, Whitfield, Omura, Fulton, and Carlson are with the Division of Nutrition, Physical Activity, and Obesity, National Center for Chronic Disease Prevention and Health Promotion, Centers for Disease Control and Prevention, Atlanta, GA, USA. Carlson is also with the Division of Population Health, National Center for Chronic Disease Prevention and Health Promotion, Centers for Disease Control and Prevention, Atlanta, GA, USA. Omura (ydk8@cdc.gov) is corresponding author. questions assessing muscle-strengthening and aerobic physical activity remained mostly unchanged during that time period. ${ }^{7,8}$

In this supplement, Whitfield et $\mathrm{al}^{9}$ used 1998-2018 NHIS data to examine trends in the prevalence of US adults who were inactive ( $<10 \mathrm{~min} / \mathrm{wk}$ of moderate-intensity equivalent physical activity), insufficiently active (10-149 $\mathrm{min} / \mathrm{wk})$, met the minimal aerobic guideline (150-300 min/wk), and met the high aerobic guideline (>300 min/wk). ${ }^{9}$ The authors observe that prevalence of inactivity decreased and meeting the high guideline increased nonlinearly across all observed subgroups throughout the monitoring period, with most statistically significant periods of change occurring after 2008. It is unknown how these patterns are influenced when the musclestrengthening guideline is included for a more comprehensive assessment of adults who meet physical activity guidelines. In addition, the most recent publication analyzing the trends in US adults meeting the muscle-strengthening guideline was limited to 11 years of NHIS data (1998-2008) and was published over a decade ago. ${ }^{10}$

To fill these gaps, we used 1998-2018 NHIS data to assess 21year trends in prevalence estimates of US adults who meet the muscle-strengthening physical activity guideline. In addition, we examined trends in meeting the combined muscle-strengthening and minimal aerobic physical activity guidelines. For both assessments, we also compared trends in meeting these guidelines across categories of selected respondent characteristics.

\section{Methods}

\section{Survey Design}

The NHIS is an annual, multistage probability sample of US households designed to be representative of the civilian, noninstitutionalized US population. The NHIS collects basic health and 
demographic information from all family members and additional information, such as information about muscle-strengthening and leisure-time physical activity, from one randomly selected adult (aged $\geq 18$ y). From 1998 to 2018, sample sizes ranged from 21,781 (2008) to 36,697 (2014) and response rates ranged from $53.0 \%$ (2017) to $74.3 \%$ (2002). ${ }^{11}$ Further information about the NHIS survey is available elsewhere. ${ }^{7,12}$

\section{Muscle-Strengthening Activities}

Respondents were asked about the number of times per week they participated in leisure-time physical activities specifically designed to strengthen their muscles, such as lifting weights or doing calisthenics. Respondents were categorized as meeting the muscle-strengthening guideline if they reported participating in muscle-strengthening activities on 2 or more times per week. ${ }^{2}$

\section{Aerobic Activity}

Respondents were asked how often and, if applicable, the duration during leisure-time they participated for at least 10 minutes at a time, in (1) vigorous-intensity activities (ie, heavy sweating or large increases in breathing or heart rate) and (2) light- or moderateintensity activities (ie, light sweating or slight to moderate increases in breathing or heart rate). To classify adults into levels of aerobic physical activity, minutes of moderate-intensity equivalent activity were calculated by counting 1 minute of vigorous-intensity activity as 2 minutes of light- or moderate-intensity activity (ie, moderate-intensity equivalent physical activity). ${ }^{2}$ Respondents were then classified into 3 activity levels: (1) active, reporting at least 150 minutes per week of moderate-intensity equivalent physical activity (ie, meeting the aerobic guideline); (2) insufficiently active, reporting some moderate-intensity equivalent physical activity but not enough to meet active definition; and (3) inactive, reporting no moderate-intensity equivalent physical activity that lasted at least 10 minutes. ${ }^{2}$ Finally, respondents who reported meeting both the muscle-strengthening and aerobic guideline were classified as meeting the combined guidelines.

\section{Selected Characteristics}

Respondents were categorized by self-reported sex (men and women), age (18-34, 35-44, 45-64, and 65+ y), race/ethnicity (white, non-Hispanic; black, non-Hispanic; Hispanic; and other, non-Hispanic), and education level (less than high school, high school, some college, and college graduate). Census region of residence (Northeast, Midwest, South, and West) was categorized based on residential address. Body mass index (BMI) was calculated from self-reported height and weight and categorized as underweight/normal $\left(<25 \mathrm{~kg} / \mathrm{m}^{2}\right)$, overweight $\left(25\right.$ to $\left.<30 \mathrm{~kg} / \mathrm{m}^{2}\right)$, and obesity $\left(\geq 30 \mathrm{~kg} / \mathrm{m}^{2}\right) \cdot{ }^{13}$

\section{Analytic Sample}

From an initial sample of 635,580 respondents, 7302 were removed for missing muscle-strengthening activity data. An additional 4235 participants $(0.7 \%)$ were missing data on education level and 20,973 $(3.3 \%)$ were missing BMI data, and were therefore excluded from estimates stratified by these variables. The analytic sample used for examining prevalence of meeting the muscle-strengthening guideline included 628,278 respondents. For analyses of meeting the combined muscle-strengthening and aerobic guideline, 14,966 respondents were excluded for missing aerobic physical activity data and 1374 were excluded for missing muscle-strengthening activity data. An additional 4100 participants $(0.7 \%)$ were missing data on education level and 20,434 (3.3\%) were missing BMI data, and were therefore excluded from estimates stratified by these variables. Therefore, the analytic sample used for analyses of meeting the combined muscle-strengthening and aerobic guidelines consisted of 619,240 respondents. For both analytic samples, missingness of physical activity data was not associated with any of the selected respondent characteristics (data not shown).

\section{Statistical Analysis}

Our study estimated the annual prevalence ${ }^{14}$ of US adults who (1) met the muscle-strengthening guideline and (2) met the combined muscle-strengthening guideline and aerobic guideline for each survey year overall and by sex, age, race/ethnicity, education level, Census region, and BMI category. Estimates were age-standardized to the 2000 US adult population using the 5 age groups. ${ }^{14}$ Prevalence of meeting the muscle-strengthening guideline was also estimated by aerobic physical activity level. In addition to meeting the combined guidelines, we also estimated the prevalence of meeting the muscle-strengthening guideline but not the aerobic (ie, musclestrengthening only), meeting the aerobic guideline but not the muscle-strengthening guideline (ie, aerobic only), and meeting neither guideline. The overall change in meeting guidelines was calculated as the difference in prevalence (in percentage points) of meeting guidelines between 2018 and 1998. Using pairwiseadjusted Wald tests with a Bonferroni correction, the difference in percentage point difference across category levels of a selected characteristic was tested to determine if changes from 1998 to 2018 differed by these subgroups. Significant linear, quadratic, and cubic trends in meeting guidelines across survey years were identified using orthogonal polynomial contrasts. If any categories within a selected characteristic displayed significant nonlinear trends in meeting guidelines, JoinPoint regression software (version 4.7; National Cancer Institute, Bethesda, MD) was subsequently used to identify the periods of greatest change by fitting trend lines with up to 4 segments (or 3 inflection points) using piecewise linear regression for all category levels of the characteristic. Apart from JoinPoint, analyses were conducted using SUDAAN (version 11.0; Research Triangle Institute, Research Triangle Park, NC) to account for the complex survey design and weighting.

\section{Results}

The prevalence of meeting the muscle-strengthening guideline among US adults increased from $17.7 \%$ in 1998 to $27.6 \%$ in 2018, with a significant linear and quadratic trend present (Figure 1). Men and women displayed similar trends across the 21 survey years, though men had consistently higher prevalence of meeting the guideline than women for each year.

Across all subgroups of the characteristics examined, the prevalence of meeting the muscle-strengthening guideline increased significantly from 1998 to 2018 (Table 1). When examining the percentage point change between 1998 and 2018, there were no significant differences in this change across categories of sex, age group, race/ethnicity, or by Census region. Respondents with a college degree and those with a high school diploma had a significantly larger increase in meeting the muscle-strengthening guideline than those with less than a high school diploma. In addition, those who were underweight/normal weight or overweight had larger increases in meeting the muscle-strengthening guideline than those with obesity, as did those who were 


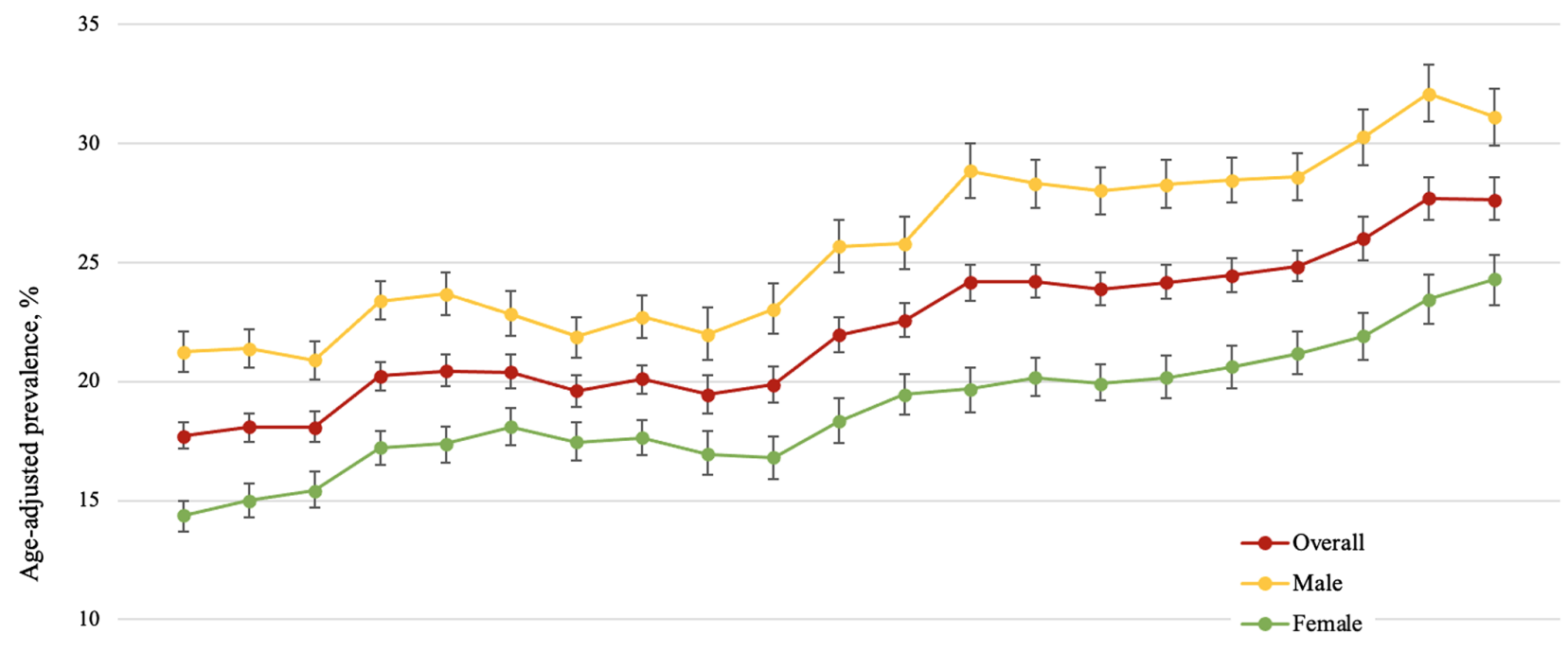

5

$\begin{array}{lllllllllllllllllllll}1998 & 1999 & 2000 & 2001 & 2002 & 2003 & 2004 & 2005 & 2006 & 2007 & 2008 & 2009 & 2010 & 2011 & 2012 & 2013 & 2014 & 2015 & 2016 & 2017 & 2018\end{array}$

Year

Figure 1 - Trends in meeting the muscle-strengthening guidelines among US adults, overall and by sex, NHIS 1998-2018. Categories for treadlines from top to bottom: males, overall, females. NHIS indicates National Health Interview Survey. Note. Significant linear and higher-order trends were present overall and by sex $(P<.05)$.

aerobically active compared with those who were inactive or insufficiently active.

All subgroups displayed significant linear trends in prevalence of meeting the muscle-strengthening guideline from 1998 to 2018, and several significant higher-order trends were also identified (Table 1). Despite identifying a significant quadratic trend using orthogonal polynomial contrasts, JoinPoint regression did not identify any significant inflection points and suggested a linear trend was best fit with an average annual percentage point change of 0.45 in meeting the muscle-strengthening guideline from 1998 to 2018 (Figure 2A). While patterns of increase varied across subgroups, there were no periods of significant decrease in meeting the guideline. For example, both non-Hispanic black and the nonHispanic other race/ethnicity group displayed no significant change from 1998 to 2005, followed by significant increases from 2005 to 2018. All subgroups except Hispanics experienced statistically significant increases in meeting the muscle-strengthening guideline in the second half of the time period observed.

The prevalence of meeting the combined muscle-strengthening and aerobic guideline increased from $14.4 \%$ in 1998 to $24.0 \%$ in 2018 with significant linear and higher-order trends present (Figure 3). The prevalence of meeting only the muscle-strengthening guideline was not significantly different over the observed timeframe (3.4\% in 1998 and 3.6\% in 2018), while the prevalence of meeting only the aerobic guideline increased from $25.7 \%$ to $30.1 \%$. Finally, the prevalence of US adults who did not meet either the muscle-strengthening or aerobic guideline decreased from $56.6 \%$ in 1998 to $42.3 \%$ in 2018 , with significant linear and quadratic trends present.

Similar to the muscle-strengthening guideline, the prevalence of meeting the combined guidelines increased significantly across all observed subgroups from 1998 to 2018 (Table 2). Patterns of increase in meeting the combined guidelines were similar to those for meeting the muscle-strengthening guideline except the difference in prevalence between 1998 and 2018 was larger among adults aged 25-34 years and 35-44 years compared with adults aged 45-64 years and 65 years or more. While the average annual percentage point change in meeting the combined guidelines was the same as the musclestrengthening guideline (0.45), the best-fitting model from JoinPoint regression indicates 4 distinct periods of change in prevalence of meeting the combined guidelines from 1998 to 2018: a significant increase from 1998 to 2002, a nonsignificant decrease from 2002 to 2006, a significant increase from 2006 to 2010, and another significant increase from 2010 to 2018 (Figure 2B). Patterns by subgroups were again similar to those for meeting the muscle-strengthening guideline, and most subgroups experienced significant increases in prevalence of meeting both guidelines from 2007 to 2018 .

\section{Discussion}

From 1998 to 2018, the age-adjusted prevalence ${ }^{14}$ of meeting the muscle-strengthening guideline among US adults increased significantly from $17.7 \%$ to $27.6 \%$. The prevalence of meeting the combined muscle-strengthening and aerobic guidelines also increased significantly from $14.4 \%$ to $24.0 \%$. For both sets of criteria, prevalence increased across all subgroups, though some increases were linear only and others exhibited both linear and higher-order trends. Most subgroups sustained significant increases in meeting the musclestrengthening and the combined muscle-strengthening and aerobic guidelines in the second half of the observed time period, specifically 2007-2018. While prevalence of meeting neither guideline decreased over time, the corresponding gains in prevalence of meeting the muscle-strengthening guideline were comprised primarily of those 


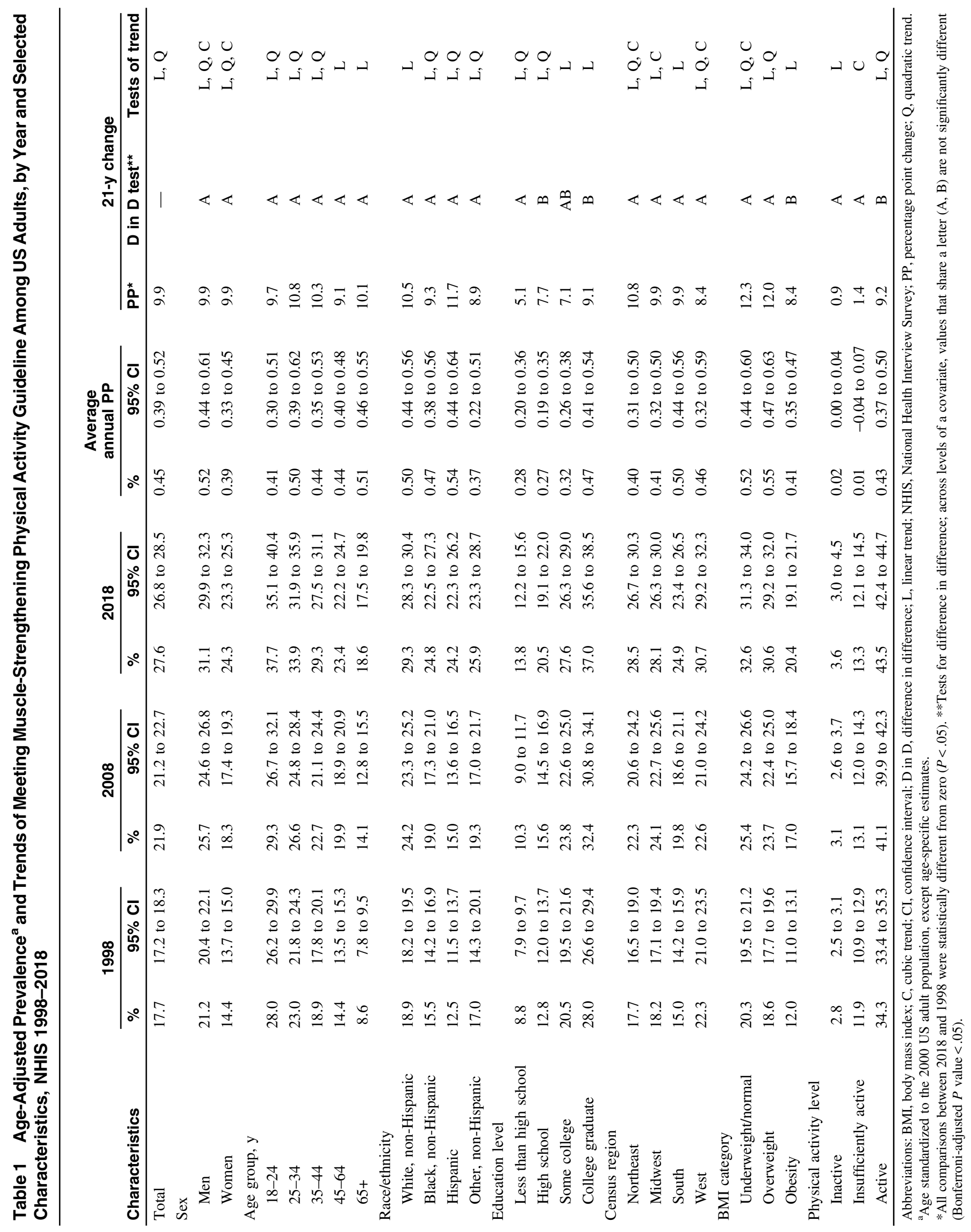


(A) Meeting the muscle-strengthening guideline

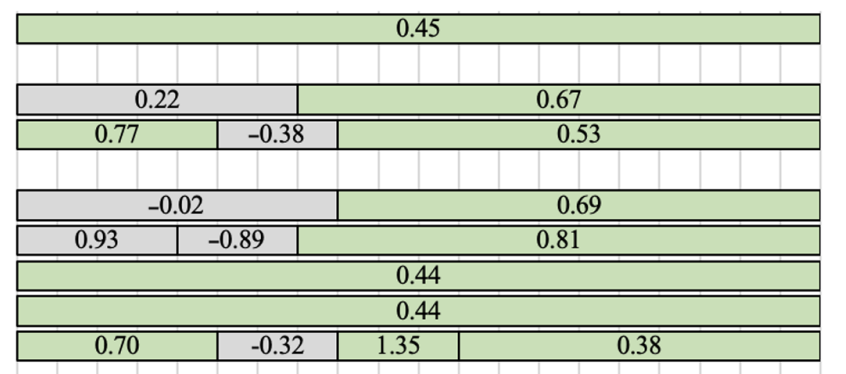

\begin{tabular}{|c|c|c|c|c|}
\hline \multicolumn{5}{|c|}{0.50} \\
\hline & & \multicolumn{3}{|c|}{0.65} \\
\hline \multicolumn{2}{|c|}{0.09} & 1.72 & 0.26 & 2.25 \\
\hline 1.20 & -1.53 & \multicolumn{3}{|c|}{0.68} \\
\hline
\end{tabular}

\begin{tabular}{|c|c|cc|}
\hline \multicolumn{2}{|c|}{0.02} & 0.49 \\
\hline \hline 0.78 & -0.72 & & 0.46 \\
\hline \hline \multicolumn{3}{|c|}{0.32} \\
\hline \hline \multicolumn{3}{|c|}{0.47} \\
\hline
\end{tabular}

\begin{tabular}{|c|c|c|c|c|}
\hline 1.37 & -0.63 & \multicolumn{2}{c|}{0.64} \\
\hline \hline \multicolumn{5}{|c|}{0.41} \\
\hline \hline \multicolumn{5}{|c|}{0.50} \\
\hline \hline \multicolumn{6}{|c|}{0.20} & 2.01 & -0.54 & 1.59 \\
\hline
\end{tabular}

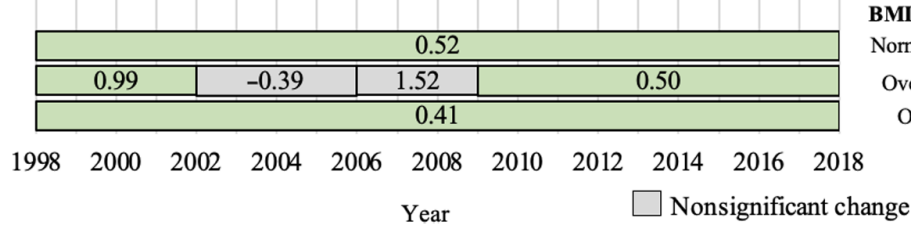

(B) Meeting both the muscle-strengthening and aerobic guideline

Overall
Sex
Men
Women
Age, y
$18-24$
$25-34$
$35-44$
$45-64$
$65+$

Race/ethnicity

White, $\mathrm{NH}$

Black, $\mathrm{NH}$

Hispanic

Other, $\mathrm{NH}$

Education level

$<$ High school
High school
Some college
College graduate

Census region

$$
\begin{aligned}
& \text { Northeast } \\
& \text { Midwest } \\
& \text { South }
\end{aligned}
$$

West

BMI category

Normal/under

Overweight

Obesity

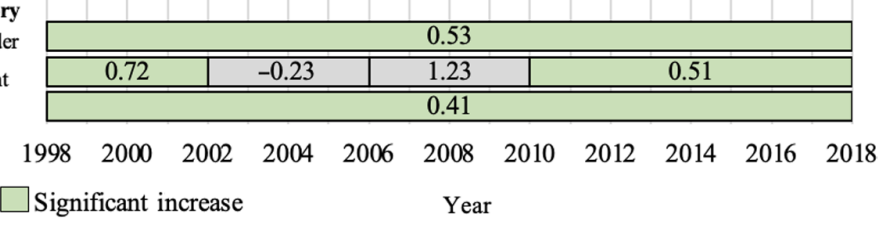

Figure 2 - Average annual percentage point change in (A) meeting the muscle-strengthening guideline ${ }^{\mathrm{a}}$ and (B) meeting both the musclestrengthening and aerobic guidelines among US adults for each segment identified using JoinPoint, NHIS 1998-2018. BMI indicates body mass index;

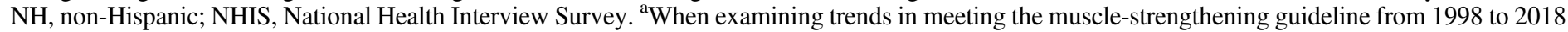
by aerobic physical activity levels, JoinPoint regression did not identify any significant inflection points for any aerobic physical activity level (ie, inactive, insufficiently active, or active).

who met the combined muscle-strengthening and aerobic guidelines. The findings from this report suggest that US adult participation in recommended levels of muscle-strengthening activities has increased over the past 2 decades, but opportunities exist to address persistent disparities in meeting the muscle-strengthening guideline, including by age and education level.

Our findings are similar to those of previous studies that assessed trends in meeting muscle-strengthening guidelines among US adults. ${ }^{10,15}$ For example, a study using data from the Behavioral Risk Factor Surveillance System found the prevalence of meeting the muscle-strengthening guideline increased significantly from $29.1 \%$ to $30.3 \%$ from 2011 to $2017 .{ }^{15}$ The differences in point estimates between the Behavioral Risk Factor Surveillance System study and the present study are likely due to several differences in methodology between the NHIS and Behavioral Risk Factor Surveillance System, such as data collection methods and survey question wording. ${ }^{8}$ However, patterns of trends in meeting musclestrengthening guidelines by sex, age, education level, and Census region were similar. In addition, a previous study estimated the prevalence of meeting the muscle-strengthening guideline from 1998 to 2008 using NHIS data. ${ }^{10}$ While our study replicated this analysis with consistent findings for the overlapping time period, it also provides a timely update of this assessment by adding another decade of available data.
In addition, our study documented trends in meeting the combined muscle-strengthening and aerobic guideline similar to previous studies. ${ }^{10,16}$ For example, a study using 2008-2017 NHIS data found an average annual percentage point increase of 0.5 , with similar patterns of estimates by age, race/ethnicity, education level, and Census region to our study. ${ }^{16}$ Despite these similarities, our study is unique in observing trends in the muscle-strengthening and combined guidelines using 21 years of survey data, taking full advantage of consistently available data over a substantial time period.

In our study, the prevalence of meeting the muscle-strengthening and combined muscle-strengthening and aerobic guidelines increased over time across all subgroups, though in many cases, disparities among subgroups were exacerbated or remained unchanged. For example, $8.8 \%$ of respondents with less than a high school education met the muscle-strengthening guideline in 1998 and the percentage point increase over the monitoring period was 5.1. Conversely, $28.0 \%$ of college graduates met the guideline in 1998 and had a percentage point increase of 9.1, resulting in a widening difference in prevalence between the least and most educated adults. Disparities have remained consistent between men and women. In 1998, men had a higher prevalence of meeting the muscle-strengthening guideline and the combined guidelines compared with women. The 21-year percentage point changes were identical for both guideline measures, indicating that this 


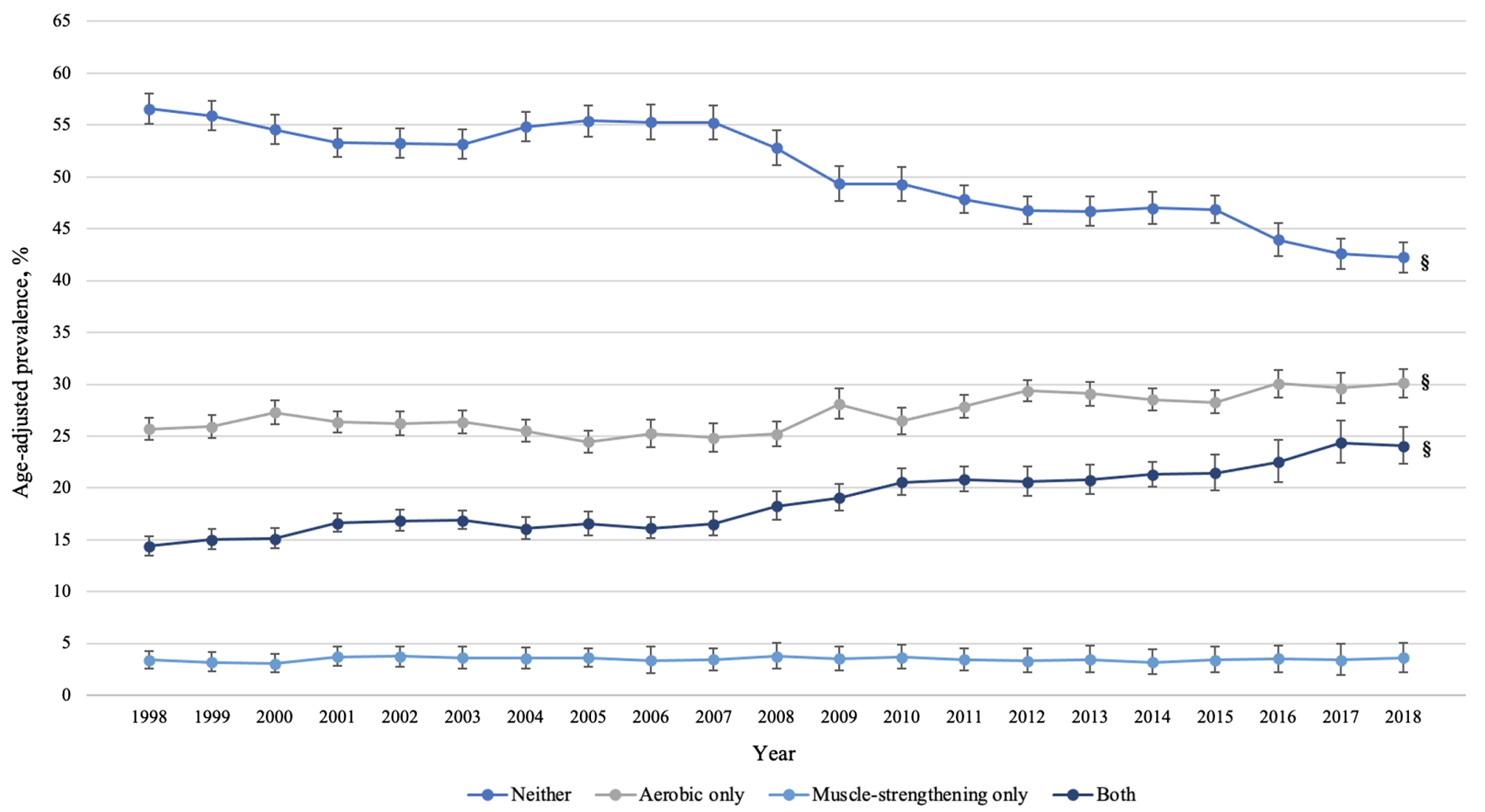

Figure 3 - Trends in meeting physical activity guidelines, alone and in combination, among US adults, NHIS 1998-2018. Categories for treadlines from top to bottom: neither, aerobic only, both, muscle-strengthening only. NHIS indicates National Health Interview Survey. ${ }^{\S}$ Significant linear and higher-order trends $(P<.05)$.

disparity persisted in 2018. Differences in meeting both guidelines also persisted throughout the observed period across age groups. Among older adults, participation in muscle-strengthening activities has specific benefits, such as improved physical functioning and ability to perform activities of daily living. ${ }^{2}$ While our study found prevalence of meeting the muscle-strengthening guideline remained consistently lowest among adults ages 65+ years throughout the monitoring period, the 21-year percentage point change in meeting the muscle-strengthening guideline was similar across all age groups. Future physical activity promotion strategies can consider focusing on priority groups, including adults with a lower education status and older adults, to help decrease disparities in muscle-strengthening activity participation.

The prevalence of meeting only the muscle-strengthening guideline did not change during the study period. In addition, while the increase in meeting the muscle-strengthening guideline was significant across all levels of aerobic activity participation, the increase was significantly larger among aerobically active respondents than among adults with no aerobic activity and adults with insufficient aerobic activity. Both findings suggest the observed overall increase in meeting the muscle-strengthening guidelines is attributable to more adults meeting both the muscle-strengthening and aerobic guidelines instead of meeting the muscle-strengthening guideline alone. Physical activity promotional strategies can take advantage of this relationship by implementing multicomponent physical activity programs that include both muscle-strengthening and aerobic physical activity components. ${ }^{2}$

Across subgroups, increases in meeting the muscle-strengthening guideline and the combined muscle-strengthening and aerobic guidelines were more prominent in the second decade of the monitoring period compared with the first decade. During the period of the present study's analysis, substantial advancement in the field of physical activity and public health occurred. For example, the first US physical activity guidelines were introduced in 2008 which included muscle-strengthening guidelines. ${ }^{1}$ The muscle-strengthening guidelines were then carried over in the second edition of the physical activity guidelines published in 2018. ${ }^{2}$ The inclusion of muscle-strengthening guidelines into national physical activity recommendations may have encouraged the incorporation of musclestrengthening activities into physical activity and public health promotional strategies nationwide, potentially helping to increase participation in muscle-strengthening activities.

Despite the observed increasing trends, point prevalence estimates suggest that most US adults still do not meet the musclestrengthening guideline. The Guidelines suggest a number of evidence-based strategies for promoting physical activity including muscle-strengthening activity. ${ }^{2}$ In addition, the Community Preventive Services Task Force recommends individually adapted health behavior change programs to increase physical activity and improve physical fitness among adults and children. ${ }^{17}$ Within the Community Preventive Services Task Force's systematic review of these programs, 7 of 9 measurements from 2 studies reported an increase in strength. ${ }^{17}$ Furthermore, evidence of programs to increase musclestrengthening activity can be indirectly measured through improved health outcomes. Some examples of evidence-based programs for older adults that incorporate muscle-strengthening exercises include EnhancedFitness (http://www.projectenhance.org/), Fit and Strong (https://www.fitandstrong.org/), and Go4Life (https://go4life.nia.nih.gov/exercises). The findings from our study can help guide future programmatic public health efforts to increase musclestrengthening participation, particularly among demographic groups where disparities exist, such as certain and age groups. 


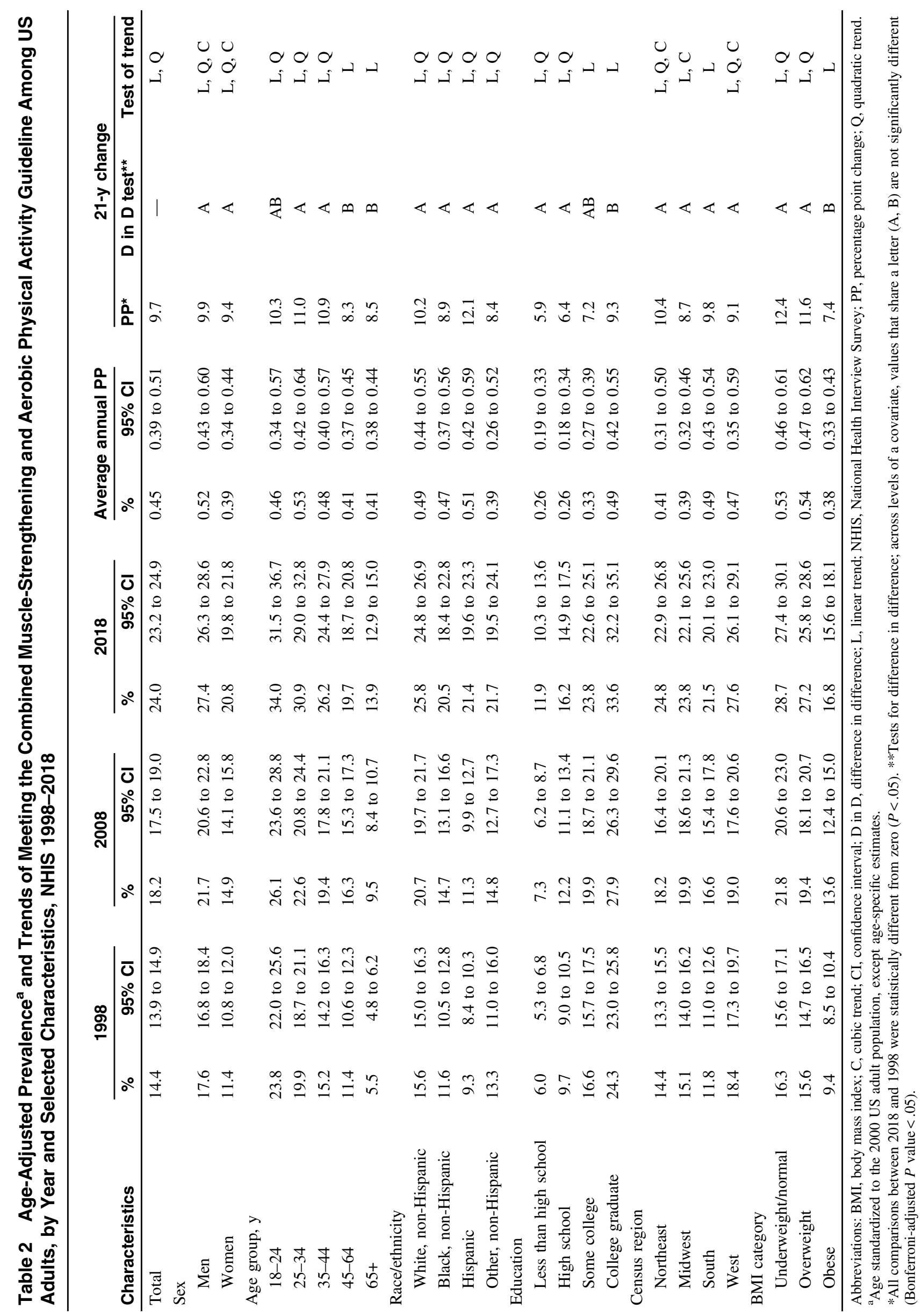


Our study has several limitations. The physical activity data used in our analyses were self-reported, which is subject to recall and social desirability biases. ${ }^{18}$ In addition, the NHIS response rates decreased over time, which may have implications for the generalizability of results during the later portion of the observation period. About $3.3 \%$ and $0.7 \%$ of the sample were missing data on education level and BMI category, respectively, which may have affected physical activity estimates within these strata. Furthermore, the NHIS asks respondents to report aerobic activity that occurred in bouts of at least 10 minutes. ${ }^{7}$ While the 2008 Physical Activity Guidelines for Americans included a bout minimum of at least 10 minutes for the adult aerobic physical activity guideline, the second edition of the guidelines removed this requirement. ${ }^{1,2}$ However, evidence suggests that eliminating the 10-minute bout minimum may have little effect on physical activity estimates. ${ }^{19}$ Finally, the second NHIS aerobic physical activity question requests that respondents report light- or moderate-intensity aerobic activity simultaneously, but the Guidelines recommend activity that is of at least moderate intensity. ${ }^{2}$ In terms of strengths, this study analyzed 21 consecutive years of consistent NHIS physical activity data, which allowed for subgroup analysis of trends in meeting physical activity guidelines across 2 decades.

\section{Conclusions}

From 1998 to 2018, the age-adjusted prevalence ${ }^{14}$ of meeting the muscle-strengthening guideline alone and the combined musclestrengthening and aerobic guidelines increased by nearly $10 \%$ points each among US adults. All subgroups displayed statistically significant increases in meeting the muscle-strengthening and combined guidelines, particularly in the second half of the monitoring period, but many disparities either persisted or were magnified. While increasing trends in prevalence of meeting the muscle-strengthening and combined guidelines are encouraging, current point prevalence estimates remain low. Continued promotion of muscle-strengthening activity using evidence-based approaches, especially those focusing on populations with persistently low participation, may help increase population levels of physical activity including participation in muscle-strengthening activities.

\section{Acknowledgment}

The findings and conclusions in this manuscript are those of the authors and do not necessarily represent the official position of the CDC.

\section{References}

1. U.S. Department of Health and Human Services. 2008 Physical Activity Guidelines for Americans. Washington, DC: Author; 2008.

2. U.S. Department of Health and Human Services. Physical Activity Guidelines for Americans, 2nd ed. 2018. https://health.gov/sites/ default/files/2019-09/Physical_Activity_Guidelines_2nd_edition.pdf

3. 2018 Physical Activity Guidelines Advisory Committee. 2018 physical activity guidelines advisory committee scientific report. 2018. https://health.gov/sites/default/files/2019-09/PAG_Advisory_Committee_ Report.pdf

4. National Center for Health Statistics. Healthy People 2010: Final Review. Hyattsville, MD: U.S. Department of Health and Human Services; 2012.
5. U.S. Department of Health and Human Services. Healthy people 2020 objective data search website: physical activity. Accessed May 5, 2020. https://www.healthypeople.gov/2020/data-search/

6. Office of Disease Prevention and Health Promotion. Healthy people 2030. https://health.gov/healthypeople. Accessed July 28, 2020.

7. National Center for Health Statistics, Centers for Disease Control and Prevention. National health interview survey: adult physical activity information. https://www.cdc.gov/nchs/nhis/physical_activity.htm. Accessed July 28, 2020.

8. Omura JD, Whitfield GP, Chen TJ, et al. Surveillance of physical activity and sedentary behavior among youth and adults in the United States: History and opportunities. J Phys Act Health. 2021;18(suppl): S6-S24. doi:10.1123/jpah.2021-0179

9. Whitfield GP, Hyde ET, Carlson SA. Participation in leisure-time aerobic physical activity among adults, National Health Interview Survey, 1998-2018. J Phys Act Health. 2021;18(suppl):S25-S36. doi:10.1123/jpah.2021-0014

10. Carlson SA, Fulton JE, Schoenborn CA, Loustalot F. Trend and prevalence estimates based on the 2008 physical activity guidelines for Americans. Am J Prev Med. 2010;39(4):305-313. PubMed ID: 20837280 doi:10.1016/j.amepre.2010.06.006

11. National Center for Health Statistics. Survey Description, National Health Interview Survey, 2018. 2019. https://ftp.cdc.gov/pub/Health_ Statistics/NCHS/Dataset_Documentation/NHIS/2018/srvydesc.pdf

12. National Center for Health Statistics. Centers for Disease Control and Prevention. National Health Interview Survey. Accessed July 28, 2020. https://www.cdc.gov/nchs/nhis/.htm

13. National Heart Lung and Blood Institute. Clinical guidelines on the identification, evaluation, and treatment of overweight and obesity in adults-the evidence report. Nat Instit Health Obes Res. 1998; 6(suppl 2):51s-209s.

14. Klein RJ, Schoenborn CA. Age adjustment using the 2000 projected U.S. population. Healthy People 2010 Statistical Notes: from the Centers for Disease Control and Prevention/National Center for Health Statistics. 2001;(20):1-10.

15. Bennie JA, Kolbe-Alexander T, Seghers J, Biddle SJH, De Cocker K. Trends in muscle-strengthening exercise among nationally representative samples of United States adults between 2011 and 2017. J Phys Activ Health. 2020;17(5):512-518. PubMed ID: 32283540 doi:10. 1123/jpah.2019-0472

16. Whitfield GP, Carlson SA, Ussery EN, Fulton JE, Galuska DA, Petersen R. Trends in meeting physical activity guidelines among urban and rural dwelling adults-United States, 2008-2017. MMWR Morb Mortal Weekly Rep. 2019;68(23):513-518. PubMed ID: 31194722 doi:10.15585/mmwr.mm6823a1

17. The Community Guide. Physical activity: individually adapted health behavior change programs. Accessed March 22, 2021. https://www. thecommunityguide.org/findings/physical-activity-individually-adaptedhealth-behavior-change-programs

18. Rzewnicki R, Vanden Auweele Y, De Bourdeaudhuij I. Addressing overreporting on the International Physical Activity Questionnaire (IPAQ) telephone survey with a population sample. Public Health Nutr. 2003;6(3):299-305. PubMed ID: 12740079 doi:10.1079/ PHN2002427

19. Ussery EN, Watson KB, Carlson SA. The influence of removing the ten-minute bout requirement on national physical activity estimates. Prev Chronic Dis. 2020;17:E19. PubMed ID: 32105588 doi:10.5888/ pcd17.190321 